Rev Biomed 2005; 16:21-27.

\title{
Infección y enfermedad por Toxoplasma gondii en animales y humanos en 23 años de observación en la provincia de Ciego de Ávila, Cuba.
}

\section{Artículo Original}

Miguel Suárez-Hernández¹, Alejandro González-Fernández², Bárbara Y. Gardón-Quirola ${ }^{3}$, Raidel MartínezSánchez .

${ }^{1}$ Centro Provincial de Higiene y Epidemiología de Ciego de Ávila, ${ }^{2}$ Facultad de Ciencias Médicas de Ciego de Ávila, ${ }^{3}$ Unidad Municipal de Higiene y Epidemiología, ${ }^{4}$ Instituto de Medicina Tropical “Pedro Kourî́. La Habana, Cuba.

\section{RESUMEN.}

La presente investigación es un diseño descriptivo, y se realizó entre 1980 y 2002 para evaluar los estudios realizados sobre Toxoplasma en animales y humanos, y así conocer la prevalencia de anticuerpos en ambos grupos y la morbilidad. Las técnicas utilizadas en animales y humanos para estudios epidemiológicos fueron intradermoreacción (toxoplasmina), reacción de fijación de complemento e inmunofluorescencia.

La mayor seropositividad en animales se detectó con la técnica de inmunofluorescencia.

No se detectaron diferencias entre los grupos poblacionales estudiados ni entre niños normales y los que tenían retraso psicomotris. En niños rurales la prevalencia fue mayor.

No existe información sobre la morbilidad animal, no así en la humana, que aunque ha existido déficit de medios diagnósticos, hay una evaluación preliminar de las afectaciones a la salud pública.

En humanos para la detección de casos se utilizaron la reacción de fijación de complemento y la inmunofluorescencia. Las causas de estudio que mayor número de enfermos aportaron fueron adenopatías, síndrome febril y oftalmopatías.

Se reportaron en el período 1998-2002 por primera vez en la etapa, casos de toxoplasmosis en pacientes VIH-SIDA.

(Rev Biomed 2005; 16:21-27)

Palabras clave: infección, enfermedad, Toxoplasma, animales y humanos.

SUMMARY.

Infection and disease by Toxoplasma gondii in humans and animals during 23 years of observation in Ciego de Ávila province, Cuba.

The aim of the present investigation is to evaluate the studies carried out on Toxoplasma in animals and humans hereby determining the prevalence of antibodies and morbidity in both groups. This study has a descriptive design and was carried out between 1980 and 2002. The techniques used on animals and

Solicitud de sobretiros: Dr. Miguel Suárez Hernández, Serafín Sánchez 155, entre Onelio Hernández y Martí, Ciego de Ávila 2

65200 Cuba. E-mail: epi.hig@trocha.cav.sld.cu

Recibido el 13/Septiembre/2004. Aceptado para publicación el 7/Enero/2005.

Este artículo está disponible en http://www.uady.mx/sitios/biomedic/revbiomed/pdf/rb051613.pdf

Vol. 16/No. 1/Enero-Marzo, 2005 


\section{Suárez-Hernández, A González-Fernández, BY Gardón-Quirola, R Martínez-Sánchez.}

humans for epidemiological studies were intradermoreaction (toxoplasmine), complement fixation reaction and inmunoflourescence.

The highest seropositivity in animals was detected using the inmunoflourescent technique.

No differences were detected among the poblation groups studied nor between normal and retarded children. The prevalence was greater among rural children.

There is no information on neither animal or human morbidity, that even though there has been a lack of diagnostic means, there is a preliminary evaluation of the afectations on public health.

Complement fixation reaction and inmunoflourescence were used to detect cases among humans. The majority of patients had the following motives for study: adenopathologies, febrile syndrome and ophthalmpathologies.

Cases of toxoplasmosis in HIV/AIDS patients were reported for the first time during the period 19982002. (Rev Biomed 2005; 16:21-27)

Key words: infection, disease, toxoplasma, animals and humans.

\section{INTRODUCCIÓN.}

La toxoplasmosis es una enfermedad protozoaria producida por el Toxoplasma gondii, el cual fue descubierto por Nicole y Manceux (1908) en un pequeño roedor llamado Ctenodactylus gondii en el norte de África (1).

El Toxoplasma gondii afecta una gama de animales domésticos, silvestres y al hombre. La infección se transmite por vía congénita y adquirida En esta última se reconoce la vía oral, en la que se adquiere por alimentos contaminados con ooquistes de Toxoplasma, y formas quísticas del parásito (2).

La pasteurización de la leche destruye el parásito. Los quistes de Toxoplasma en la carne pueden sobrevivir a más de $40^{\circ} \mathrm{C}$ hasta 3 semanas. Los mismos mueren si la carne se ha conservado congelada a menos de $15^{\circ} \mathrm{C}$ durante más de tres días o a menos de $20^{\circ} \mathrm{C}$ durante más de dos días. La carne que se ha calentado intensamente hasta una temperatura de $65^{\circ} \mathrm{C}$ y mantenido dicha temperatura durante 4 ó 5 minutos o más, suele contener quistes viables. El salado y ahumado destruyen los Toxoplasmas (3).

Se han descrito brotes por la ingestión de leche de cabra no pasteurizada (4) y se han aislado Toxoplasmas en carnes y sus derivados (5). Se describe, por algunos autores, que la toxoplasmosis se puede adquirir por transfusiones sanguíneas en pacientes inmunocomprometidos (6).

La entidad en los animales puede ser asintomática y sintomática, presentándose en forma aislada o brotes localizados (7).

Para el diagnóstico seroepidemiológico se han efectuado diversas técnicas como la intradermoreacción, fijación de complementos, inmunofluorescencia y ELISA (8).

En la Provincia Ciego de Ávila, Cuba, se han publicado diferentes investigaciones sobre Toxoplasma. En España se reportaron 2 casos de Toxoplasmosis congénita (9), y en Colombia se publicó la morbilidad conocida durante años de seguimiento (10).

El presente estudio abordará las investigaciones realizadas en animales y humanos en 23 años de observación.

\section{MATERIALESY MÉTODOS.}

El presente diseño es un estudio descriptivo y abarca las investigaciones realizadas sobre Toxoplasma gondii en la provincia de Ciego de Ávila entre 1980 y 2002.

En el área de salud pública el diagnóstico se realizó sistemáticamente. Entre 1980 y 1983 la prueba utilizada fue la toxoplasmina para pesquizaje epidemiológico. Para el diagnóstico de la enfermedad de 1984 a 1987 se empleó la reacción de fijación de complemento de Toxoplasma (R.F.C). Mientras que entre 1989 y 2002 se utilizó la inmunofluorescencia indirecta de Toxoplasma (I.F.I).

En el área de salud animal no se ha realizado diagnóstico sistemático sino investigaciones puntuales. La toxoplasmina utilizada procedía de los laboratorios Sevac de Praga de la República Checa. A cada animal se inoculó $0.1 \mathrm{ml}$ de antígeno por vía intradérmica e

\section{Revista Biomédica}




\section{Toxoplasmosis en Cuba}

igual dosis del control variando la región anatómica de aplicación según la especie. La lectura de la intradermoreacción se efectuó a las 48 horas, considerándose positivo el que tuviera una pápula mayor de $5 \mathrm{~mm}$.

Los pesquisajes con toxoplasmina se realizaron en 2241 animales aparentemente sanos, desglosados en 1154 cerdos, 284 equinos, 259 conejos, 200 gallinas, 100 ovinos, 79 perros, 54 caprinos, 49 gatos, 37 patos y 25 pavos.

Se estudiaron 427 personas aparentemente sanas de diferentes grupos ocupacionales, así como 162 niños con retraso psicomotor y 110 supuestamente sanos. En total se investigaron con la intradermoreacción 699 personas. En los humanos se inocularon también $0.1 \mathrm{ml}$ de antígeno por vía intradérmica en la cara anterior del brazo. Se consideró positivo a una pápula mayor de $5 \mathrm{~mm}$.

La R.F.C. se trabajó también con un antígeno checo. Se estudiaron con la reacción de fijación de complemento (R.F.C.) 2992 animales desglosados en 2189 porcinos, 485 ovinos, 145 equinos, 86 caninos, 60 conejos y 27 gatos. Se consideró seropositivo el que tuviera un título de 18 o más.

La inmunofluorescencia indirecta de Toxoplasma se realizó con láminas y conjugados elaborados en el centro nacional de biopreparados. La inmunofluorescencia de Toxoplasma se efectuó en 1958 animales de ellos, 1473 bovinos y 485 ovinos. Se consideró positivo al que tuviera un título de 132 o más. La muestra fue calculada y es representativa de ambas poblaciones.

Se investigaron con esta técnica 548 niños de escuelas primarias y secundarias de áreas urbanas (253) y rurales (295).

En el presente estudio se analizaron los pacientes estudiados para descartar toxoplasmosis según las diferentes causas. Se consideró estudiado a la persona que se realizó dos muestras. Se investigaron en la etapa 7210 personas. Entre 1984 y 1987 se empleó la R.F.C. de Toxoplasma, mientras que entre 1989 y 2002 se utilizó la I.F.I.

Se consideró caso confirmado de toxoplasmosis por la técnica de reacción de fijación de complemento
R.F.C., aquella persona que en el suero pareado tuviera seroconversiones oincremento de título de dos o más diluciones o en el primer suero tuviera una dilución de 1128 o más.

En la inmunofluorescencia se aceptaron también los mismos principios de la R.F.C. en lo referente a seroconversiones o incremento de títulos de dos o más diluciones. Además, si el primer suero tenia un titulo de 1512 o más.

Se refleja en el estudio 322 casos confirmados desglosados en niños y adultos.

Se usaron los métodos estadísticos de Chi cuadrado y test de Fischer para analizar las diferencias entre las categorías y especies.

\section{RESULTADOS.}

En el cuadro 1 aparecen las investigaciones realizadas en las diferentes especies de animales con la técnica de toxoplasmina. El $6 \%$ fueron positivas, la mayor frecuencia se detectó en caprinos, conejos y gallinas. Se detectó que hay diferencias en la reactividad entre especies $(\mathrm{P}<0.01)$.

El $29 \%$ de los animales estudiados con la R.F.C.

\section{Cuadro 1}

Investigación con toxoplasmina en las diferentes especies de animales.

\begin{tabular}{|c|c|c|c|}
\hline Especies & Investigados & Positivo & $\%$ \\
\hline Cerdos & 1154 & 16 & 1 \\
\hline Equinos & 284 & 15 & 5 \\
\hline Conejos & 259 & 44 & 17 \\
\hline Gallinas & 200 & 32 & 16 \\
\hline Ovinos & 100 & 3 & 3 \\
\hline Perros & 79 & 3 & 4 \\
\hline Gatos & 49 & 2 & 4 \\
\hline Caprinos & 54 & 10 & 18 \\
\hline Patos & 37 & 2 & 5 \\
\hline Pavos & 25 & - & - \\
\hline Total & 2241 & 127 & 6 \\
\hline
\end{tabular}

Chi Cuadrado $175.10 \quad p<0.01$

Test de independencia $x^{2} \quad p<0.05$

Probabilidades de las celdas mayores que el valor de la distribución marginal por la columna de los positivos. Fuente: Departamento provincial de epidemiología Nota: Los porcientos están ajustados.

Vol. 16/No. 1/Enero-Marzo, 2005 
M Suárez-Hernández, A González-Fernández, BY Gardón-Quirola, R Martínez-Sánchez.

\section{Cuadro 2}

Resultados obtenidos con la reacción de fijación de complemento (R.F.C) en diferentes especies animales.

\begin{tabular}{lccc}
\hline Especies & Números & Seropositivo & \% \\
\hline Porcinos & 2189 & 305 & 14 \\
Ovinos & 485 & 390 & 80 \\
Equinos & 145 & 90 & 62 \\
Caninos & 86 & 40 & 46 \\
Conejos & 60 & 28 & 47 \\
Felinos & 27 & 25 & 92 \\
Total & 2992 & 879 & 29 \\
\hline
\end{tabular}

Chi cuadrado $998.05 \quad \mathrm{p}<0.01$

Fuente: Departamento provincial de epidemiología Nota: Los porcientos están ajustados.

fueron seropositivos, existiendo también diferencias entre las especies evaluadas ( $\mathrm{P}<0.01)$ (cuadro 2).

El $65 \%$ de los animales pesquisados con la inmunofluorescencia de Toxoplasma, tuvieron anticuerpos, variando la proporción entre bovinos y ovinos $(\mathrm{P}<0.01)$ (cuadro 3).

En los diferentes grupos humanos estudiados con toxoplasmina la reactividad fue del $24 \%$, no existiendo diferencias entre las proporciones ( $\mathrm{P}>0.05)$ (cuadro 4).

El $14 \%$ de los niños estudiados con la toxoplasmina fueron reactivos, siendo la reactividad mayor en los pacientes con retraso psicomotor sin llegar al umbral de la diferencia estadística (cuadro 5). El $43 \%$ de los niños de escuelas primarias y secundarias estudiados tenían la infección toxoplásmica, siendo mayor la frecuencia en el área

\section{Cuadro 3}

Resultados obtenidos con la inmunofluorescencia de Toxoplasma en bovino y ovino.

\begin{tabular}{lccc}
\hline Especies & Investigados & Seropositivos & $\%$ \\
\hline Bovino & 1473 & 882 & 60 \\
Ovino & 485 & 390 & 80 \\
Total & 1958 & 1272 & 65 \\
\hline
\end{tabular}

Chi cuadrado: $64.03 \quad \mathrm{p}<0.01$

Fuente: Departamento de epidemiología

Nota: Los porcientos están ajustados.

\section{Cuadro 4}

Grupos pesquisados con toxoplasmina y la reactividad.

\begin{tabular}{lccc}
\hline \multicolumn{1}{c}{ Grupos } & Estudiados & Reactivos & \% \\
\hline $\begin{array}{l}\text { Ama de casa } \\
\text { Trabajadores de }\end{array}$ & 106 & 27 & 25 \\
$\begin{array}{l}\text { pasteurizadora } \\
\text { Administrativos }\end{array}$ & 94 & 18 & 19 \\
$\begin{array}{l}\text { Veterinarios y } \\
\text { carniceros }\end{array}$ & 79 & 23 & 29 \\
$\begin{array}{l}\text { Trabajadores de } \\
\text { servicios }\end{array}$ & 70 & 18 & 23 \\
Total & 427 & 19 & 27 \\
\hline
\end{tabular}

Chi cuadrado $2.50 \quad$ p> 0.05

Fuente: Departamento provincial de epidemiología. Nota: Los porcientos están ajustados.

rural $(\mathrm{P}<0.01)$. (cuadro 6).

El $4 \%$ de los pacientes estudiados tenían toxoplasmosis. La mayor frecuencia de enfermos en patologías que tienen un buen nivel de investigación fueron las adenopatías, síndrome febril y oftalmopatías. De los 322 casos confirmados 319 eran inmunocompetentes y 3 inmunodeprimidos (pacientes VIH-SIDA) (cuadro 7). El 64\% de los enfermos fueron adultos y el $36 \%$ niños. En los primeros predominaron los masculinos y en los segundos los femeninos. (cuadro 8). El 66\% de los casos se confirmaron por la inmunofluorescencia indirecta de Toxoplasma y el $34 \%$ por la RFC (cuadro 9).

\section{DISCUSIÓN.}

El presente estudio ha demostrado la amplia circulación que tiene el Toxoplasma gondii en las

\section{Cuadro 5}

Toxoplasmina inoculada a niños normales y con retrasos psicomotor.

\begin{tabular}{lccl}
\hline \multicolumn{1}{c}{ Grupos } & Estudiados & Reactivos & \% \\
\hline Normales & 110 & 13 & 12 \\
Retraso psicomotor & 162 & 26 & 16.3 \\
Total & 272 & 39 & 14 \\
\hline
\end{tabular}

Chi cuadrado $0.95 \quad \mathrm{p}>0.05$

Fuente: Departamento provincial de epidemiología. Nota: Los porcientos están ajustados.

\section{Revista Biomédica}


Toxoplasmosis en Cuba

Cuadro 6

Análisis de la positividad a Toxoplasma con la técnica de inmunofluorescencia en niños según la procedencia.

\begin{tabular}{lccc}
\hline Procedencia & Investigadas & Seropositivas & \% \\
\hline Urbana & 253 & 69 & 27 \\
Rural & 295 & 168 & 57 \\
Total & 548 & 237 & 43 \\
\hline
\end{tabular}

Chi cuadrado $33.26 \quad \mathrm{p}<0.01$

Fuente: Departamento provincial de epidemiología.

Nota: Los porcientos están ajustados.

muestras estudiadas.

Los pesquisajes realizados en bovinos y ovinos con la técnica de inmunofluorescencia son representativos de la seroprevalencia en estas especies. El 80\% de los ovinos estudiados fueron positivos, se ha demostrado que esta especie juega un papel importante en la transmisión de la enfermedad al hombre ya que los quistes se mantienen durante mucho tiempo, a diferencia del bovino que juega un papel secundario ya que sus quistes persisten poco tiempo (11).

La mayor detectabilidad de anticuerpos por las técnicas de reacción de fijación de complemento e inmunofluorescencia en relación a la toxoplasmina se deben a la mayor sensibilidad de aquellas (12). En la provincia el Instituto de Medicina Veterinaria no cuenta con medios diagnósticos para el estudio de Toxoplasma. Las investigaciones realizadas en

\section{Cuadro 7}

Causas de estudios de Toxoplasmas y casos confirmados.

\begin{tabular}{lccc}
\hline $\begin{array}{l}\text { Causas de } \\
\text { Estudio }\end{array}$ & $\begin{array}{c}\text { Casos } \\
\text { Investigados }\end{array}$ & $\begin{array}{c}\text { Casos } \\
\text { Confirmados }\end{array}$ & $\%$ \\
\hline Embarazadas & 2620 & 51 & 2 \\
Abortadoras & 1958 & 57 & 3 \\
Adenopatías & 1662 & 150 & 9 \\
Oftalmopatías & 639 & 37 & 6 \\
Síndrome febril & 265 & 23 & 9 \\
Anomalías congénitas & 59 & 1 & 2 \\
VIH-SIDA & 8 & 3 & 37 \\
Total & 7210 & 322 & 4 \\
\hline
\end{tabular}

Fuente: Departamento provincial de epidemiología

\section{Cuadro 8}

Toxoplasmosis en niños y adultos por sexo.

\begin{tabular}{lccccccc}
\hline Categoría & Masculino & $\%$ & Femenino & $\%$ & Total & $\%$ \\
\hline Niños & 86 & 60 & 31 & 17 & 117 & 36 \\
Adultos & 57 & 40 & 148 & 83 & 205 & 64 \\
Total & 143 & 100 & 179 & 100 & 322 & 100 \\
\hline
\end{tabular}

Fuente: Departamento provincial de epidemiología.

Nota: Los porcientos están ajustados.

animales en el presente estudio se efectuaron por el centro provincial de higiene y epidemiología y estuvieron encaminadas a detectar infección pero no la enfermedad. Por lo cual no se conocen las afectaciones a la salud animal que provoca esta zoonosis parasitaria. Existen reportes en la literatura que el Toxoplasma gondii es causa de abortos y crías muertas en ovejas y cerdos así como puede provocar cuadros neurológicos en terneros (13 y 14).

En las personas no se detectaron diferencias en el pesquizaje de diferentes grupos ocupacionales. Esta entidad no es una enfermedad profesional. En Canadá no obstante se señala que los que trabajan con animales: matarifes, carniceros, cocineros, agricultores, jardineros y trabajadores de laboratorio tienen el mayor riesgo de enfermar (15).

La mayor reactividad en niños con retraso psicomotor que en niños sanos, pudiera deberse a una disminución de la higiene como consecuencia de su problemática.

El $43 \%$ de seroprevalencia de anticuerpos

\section{Cuadro 9}

Casos confirmados de toxoplasmosis según técnica empleada.

\begin{tabular}{lcc}
\hline Técnica Empleada & Casos Confirmados & $\%$ \\
\hline $\begin{array}{l}\text { Reacción de fijación de } \\
\text { complemento (R.F.C) }\end{array}$ & 111 & 34 \\
$\begin{array}{l}\text { Inmunofluorescencia } \\
\text { indirecta de toxoplasma }\end{array}$ & & \\
(IFI) & 211 & 66 \\
Total & 322 & 14 \\
\hline
\end{tabular}

Fuente: Departamento provincial de epidemiología. Nota: Los porcientos están ajustados. 


\section{Suárez-Hernández, A González-Fernández, BY Gardón-Quirola, R Martínez-Sánchez.}

detectados en niños escolares es representativo de la población de niños de escuelas primarias en la provincia. La mayor proporción en niños rurales pudiera deberse a los siguientes factores:

a) Menor higiene

b) Mayor facilidad de exposición a las fuentes contaminantes y las vías de transmisión.

En la provincia Ciego de Ávila se realizó un estudio de prevalencia y se encontró el $55 \%$ (16).

El sexo femenino predominó en los adultos con toxoplasmosis. Se ha demostrado que las mujeres al participar en las actividades culinarias tienen un riesgo de adquirir la enfermedad (17). La mayor frecuencia de niños de sexo masculino con toxoplasmosis pudiera deberse a una mayor movilidad y por tanto mayor contacto con el medio ambiente.

La adenopatía fue la causa que mayor frecuencia de casos aportó. La literatura revisada expresa que la toxoplasmosis adquirida más frecuente es la ganglionar y de mayor incidencia en niños y jóvenes (18). En una casuística en niños, el $70.3 \%$ de los casos era forma ganglionar y el $25 \%$ oculares. Se observó un predominio de 2:1 del sexo masculino sobre el femenino (19). La baja frecuencia de toxoplasmosis congénita en este estudio se debe a que no existe un pesquisaje sistemático. Se ha estimado que la frecuencia de toxoplasmosis congénita oscila entre 0.5 y 1 por cada 1000 nacidos vivos (20).

En el quinquenio de 1998 al 2002 se confirmaron tres casos SIDA con toxoplasmosis. En Cuba el Toxoplasma gondii es un importante oportunista y se ha demostrado que su presencia esta vinculada a una disminución de los linfocitos T CD4 (21).

Aunque no se trabajaron los casos simultáneamente con la reacción de fijación de complemento y la inmunofluorescencia de toxoplasma, se conoce que esta técnica tiene mayor detectabilidad (22). Eso explica la mayor frecuencia en enfermos diagnosticados con este método.

\section{CONCLUSIONES.}

1.- La mayor frecuencia de seropositivos en los

\section{Revista Biomédica}

pesquisajes epidemiológicos realizados con animales fue con la técnica de inmunofluorescencia de Toxoplasma.

2.- No hay información sobre la morbilidad en los animales.

3.- No se detectaron diferencias en la presencia de anticuerpos de Toxoplasma en los grupos ocupacionales estudiados.

4.- No se detectaron diferencias entre niños normales y con retraso psicomotor en la inyección toxoplásmica 5.- Fue mayor la seropositividad en niños rurales que urbanos.

6.- En los casos de toxoplasmosis, predominó en los niños el sexo masculino y en los adultos el femenino.

7.- Las causas de estudio que mayor positividad aportaron fueron adenopatías, síndrome febril y oftalmopatías. La técnica de inmunofluorescencia detectó mayor cantidad de enfermos que la reacción de fijación de complemento.

\section{RECOMENDACIONES:}

1.- Montar en el laboratorio provincial del instituto de medicina veterinaria el diagnóstico de Toxoplasma.

2.- Implantar el programa de zoonosis parasitaria.

\section{REFERENCIAS.}

1.- Mombro M. Congenital toxoplasmosis 10 years follows Eur J Pediatr 1995; 154: 635-9.

2.- Mittal V, Bhatia R, Schgal S. Prevalence of Toxoplasma antibodies among woman with $\mathrm{BOH}$ and general population in Delhi. J Communic Dis 1990; 22: 223-6.

3.- Thiermann E, Atlas A, Loren M. Apreciaciones sobre el riesgo relativo de la transmisión sanguínea. Parasitología al día 1988; 12 (2): 81-83.

4.- Chiari H, Pereira D. Toxoplasmosis humana adquirida a través de ingestión de leche de cabra. Mem Inst Oswaldo Cruz 1984; 79: 337-40.

5.- Almeida M, Magalhaes C, Ferreira L, Moraes P, Pezati N, Neres R. Isolamento de Toxoplasma gondii de carnes y derivados provenientes de región endémica de Toxoplasmosis ocular. Arch Bras Oftalmol 1990; 53:60-6. 


\section{Toxoplasmosis en Cuba}

6.- Dubey JP, Thulliez P, Powell EC. Toxoplasma gondii in lowa Sows comparision of antibody titers to isolation of Toxoplasma gondii by bioassaayss in mice and cats. J Parasitol 1995; 81: 48-53.

7.- Aguilar F. Parasitología medica, 2da edición. Ciudad de Guatemala: Editorial Delgado; 1991.p. 281-91.

8.- Zemburowa K, Roczniark M, Sokolowska B. The comparision of serological test used in diagnosing active toxoplasmosis. Wiad Parazitol. 1990; 36:121-7.

9.- Suárez M. Presentación de los dos primeros casos de toxoplasmosis congénita en la provincia Ciego de Ávila, Cuba. Rev Esp Pediatr 1998; 54: 271-4.

10.- Suárez M. Toxoplasmosis en la provincia de Ciego de Ávila, Cuba. Rev Epidem Antioquia 1998; 23: 67-74.

11.- Morillo N, Bonjante G. Primer hallazgo de Toxoplasma gondii en un bovino del estado de Yaracuy, Venezuela. Kasmera 1994; 22: 71-8.

12.- Rivera F, Calderón E, Olivera J, Conde J, Echamiz G. Comparación de tres pruebas serológicas para el diagnostico de la toxoplasmosis. Infectología 1988; 8: 127-30.

13.- Skinner L J, Timperley AC, Wightman D, Chatterton JM, Ho Yen Do. Simultaneous diagnosis of toxoplasmosis in goats and goatower is family. Scand J Infect Dis 1990; 22: 359-61.

14.- Dubey J P. Duration of Inmunity to shedding of Toxoplasma gondii oocysts by cat, J Parasitol 1995; 81: 4105.

15.- Centre for ocupational health and safety. Toxoplasmosis: A summary of ocupational health concern. Edited by Canadian Centre for occupational health and safety. Hamilton; 1989. p. 1-3.

16.- Martínez R, Machin R, Fachado A, Pividal J, Cruz R, Suárez M. Algunos resultados sobre una encuesta de Toxoplasma. Investigación clínica 1991; 32: 13-26.

17.- Lappalalnen M. Cost- benefit analysis of screening for toxoplasmosis during pregnancy. Scand J infects Dis 1995; 27:265-72.

18.- Mole J R, Suárez D, Alonso F, Piquera N. Lo que hacemos para prevenir la toxoplasmosis congénita en Manzanillo. Rev Cub Med Gen Integr 1992; 8: 330-2.
19.- Villalonga J F, Antonide Jogma P S, García C. Estudio de pacientes pediátricos con toxoplasmosis. Ciencias Médicas. San Miguel de Tucuman 1986; 1: 37-47.

20.- Mombro M. Congenital toxoplasmosis 10 years follow Eur J Pediatr 1995; 154: 635-9.

21.- Naloska T, Cariatfu T. Toxoplasmosis is caused of sensoneural. Mem Inst Oswaldo Cruz 1994; 89: 485-90.

22.- Russo M, Galanti B. Prevention of congenital toxoplasmosis. Clínica Terapéutica Roma 1990; 134: 383-92. 